eventually detecting a spuriously significant treatment difference (type I error). Accordingly, it is good practice to decide in advance precisely what would be sufficiently strong evidence of a treatment effect to merit stopping the trial, taking into account the magnitude of the difference in outcome considered clinically important, the number of interim analyses to be performed, and the level of statistical significance required (Pocock, 1983). Unfortunately, Bisson et al do not state whether any stopping rules were explicitly determined before the trial commenced, or whether there was any limit on the number of interim analyses, and it is therefore possible that their study was prematurely stopped at an inappropriate point.

This difficulty would be less important were it not that at the time at which the study was terminated the debriefed group not only had experienced more severe burn traumas than the controls, but in addition almost twice as many debriefed subjects had reported significant previous trauma, both of these factors increasing their risk for the development of more numerous and severe post-traumatic symptoms (McFarlane \& Yehuda, 1996). It may be that had the study continued recruiting patients according to its random protocol the background differences between the two groups would have diminished and the outcome findings could have been different.

Bisson, J. I., Jenkins, P. L., Alexander, J., et ol (1997) Randomised controlled trial of psychological debriefing for victims of acute burn trauma. British journal of Psychiotry. 171 . 78-81.

Hobbs, M., Mayou, R., Harrison, B., et ol (1996)

A randomised controlled trial of psychological debriefing for victims of road traffic accidents. British Medical journal, 313. 1438-1439.

McFartane, A. C. \&ohuda, R. (1996) Resilience. vulnerability, and the course of posttraumatic reactions. In Traumatic Stress (eds. B. A. van der Kolk. A. C. McFarlane \& L. Weisaeth). pp. 155-181. London: Guilford Press.

David Reiss, Morven Leese Department of

Forensic Psychiatry, Institute of Psychiatry, De Crespigny Park, London SE5 8AF

\section{Cost-effectiveness of clozapine}

Sir: Robert \& Kennedy's editorial (1997) on our paper (Aitchison \& Kerwin, 1997) cannot pass without comment. This was not a clinical trial of clozapine and should not be judged as such: our study was a costeffectiveness analysis.
However, we would like to comment on the efficacy figure that they quoted. They quote Baldessarini \& Frankenburg (1991) as giving a figure of $13 \%$ of patients being better off on clozapine than on typical neuroleptics (from trials, largely double-blind), omitting a comment from the same paper that "it is increasingly apparent that . . . about a third of patients with chronic psychosis improve more in response to clozapine than other drugs". In a more recent review, Umbricht et al (1995) concluded that "clozapine is the first antipsychotic agent with proven superiority over conventional antipsychotics in the treatment of severely ill, chronic schizophrenic patients", showing a response rate $26-46 \%$ higher than that to chlorpromazine or haloperidol. The Cochrane Collaboration Schizophrenia Group systematic review (Wahlbeck et al, 1997) on clozapine currently includes 27 randomised controlled trials, and concludes that clozapine is "convincingly more effective than 'typical' neuroleptic drugs in reducing symptoms of schizophrenia, producing clinically meaningful improvements and postponing relapse".

In the UK those who are eligible for clozapine are refractory to, or intolerant of, standard neuroleptics. The response rate of such patients to standard neuroleptics is therefore very low (the argument is circular). Randomised controlled trials are hardly necessary to conclude that there is a substantial advantage of clozapine over standard neuroleptics in treatment-resistant schizophrenia in terms of efficacy.

Aitchison, K. \&orwin, R. W. (1997) Cost-effectiveness of clozapine. British Journal of Psychiotry, 17I, 125-130.

Baldesearini, R. J. \& Frankenburz, F. R. (1991) Clozapine. A novel antipsychotic agent. New England fournal of Medicine, 324. 746-754.

Robert, G. Kennedy, P. (1997) Establishing costeffectiveness of atypical neuroleptics. British journal of Psychiotry, 171, 103-104.

Umbricht, D. S. G., Lieberman, J. A. \& Kane, J. M. (1995) The clinical efficacy of clozapine in the treatment of schizophrenia. Reviews in Contemporary Phormocotherapy, 6. 165-186.

Wahbeck, K., Cheine, M., Essall, M. A., et of (1997) Clozapine for schizophrenia. Clozapine versus 'typical' neuroleptic medication for schizophrenia. In Schizophrenio Module (eds C. E. Adams, L. Duggan, M. J. De Jesus, et al). The Cochrane Database of Systematic Reviews (available in the Cochrane Library). London: BMJ Publishing.

R.W. Kerwin, K. J. Aitchison Section of Clinical Neuropharmacology, Institute of Psychiatry, London SE5 8AF

\section{Cannabis and schizophrenia}

Sir: In their recent editorial Hall \& Solowij (1997) were perhaps a little too sanguine about the relationship between cannabis consumption and schizophrenia. At least six studies, several of them prospective, have found a strong relationship between levels of cannabis use before the onset of psychotic symptoms and the subsequent development of schizophrenia and other chronic psychotic illnesses (Boutros \& Bowers, 1996). Moreover, those patients with schizophrenia who had used cannabis prior to the onset of their illness are distinct, both demographically and clinically, from those who had not: younger, more often male, with better premorbid functioning, and a different symptom pattern, response to neuroleptics and subsequent disease course (Longhurst et al, 1997). Thus, prolonged cannabis use appears to induce chronic psychosis in a group of individuals who are sufficiently different from the general mass of patients with schizophrenia to suggest that, in the absence of such use, they might not be especially vulnerable.

For many years, clinicians have been aware that the use of cannabis may lead to persistent psychosis (Glass \& Bowers, 1970). The weight of both decades of clinical experience and the current literature strongly suggests that cannabis use can result in chronic psychoses, including schizophrenia.

Boutros, N. N. \& Bowers, M. B. (1996) Chronic substance-induced psychotic disorders: the state of the literature. Journal of Neuropsychiotry and Clinical Neurosciences, 8, 262-269.

Glass, D. S. \& Bowers, M. B. (1970) Chronic psychosis associated with long-term psychotomimetic drug use. Archives of General Psychiotry, 23, 997-1003.

Hall, W. \& Solowij, N. (1997) Long-term cannabis use and mental health. British Journal of Psychiotry, 17I, 107-108.

Longhurst, J. G., Boutros, N. N. \& Bowers, M. B. (1997) Cannabis-induced chronic psychosis - an underacknowledged disonder? Australion and New Zealand fournal of Psychiatry, 31, 305-306.

J. G. Longhurst Department of Psychiatry and Connecticut Mental Health Center, Yale University School of Medicine, New Haven, CT 06519, USA

Author's reply: It is difficult in the space limits of an editorial to do justice to the issues involved in deciding whether cannabis use can produce chronic psychoses such as schizophrenia, when informed opinion differs and the evidence is inconsistent. 
For example, Hambrecht \& Hafner's (1996) study of 232 first-episode cases of schizophrenia reported large and consistent differences in age at onset between those who misused alcohol and other drugs and those who did not. The age at onset of the first psychotic symptoms was 6-8 years earlier in drug users and 1-4 years earlier in those who misused alcohol. They also found that alcohol and drug misusers had more positive psychotic symptoms and more social problems than those who had not misused drugs.

These results differ from those of Kovasznay et al's (1993) study of substance misuse and its correlates among newly incident cases of psychosis. They found similarly high rates and patterns of substance misuse among their patients with schizophrenia ( $n=76$ ): $50 \%$ of males and $17 \%$ of females had a history of substance misuse disorders, with alcohol and cannabis being the most commonly misused drugs. However, they did not find any differences in age at onset of psychotic disorders between those who had and those who had not misused drugs, and there was no suggestion that a difference went undetected because of low statistical power. Similar results emerged from Cuffel et al's (1993) analysis of substance misuse among 231 cases of schizophrenia identified in the Epidemiologic Catchment Area study. Substance misusers in this study did not differ from non-misusers in age at onset, treatment history or the prevalence of psychotic symptoms.

In the face of these conflicting results it is difficult to decide upon the nature of the relationship, if any, between cannabis use and the age at onset, premorbid personality, or the prevalence of psychotic symptoms in people with schizophrenia. None of this exculpates cannabis use from being a possible risk factor for psychosis, or its continued use as a behaviour that may exacerbate the course of schizophrenia.

Cuffel, B. J., Helthoff, K. A. \& Lawson, W. (1993)

Correlates of patterns of substance abuse among patients with schizophrenia. Hospital and Community Psychiatry. 44. 247-251.

Hambrecht, M. Mafner, H. (1996) Substance abuse and the onset of schizophrenia. Biological Psychiatry, 40, 1155-1163.

Kovaeznay, B., Bromet, E., Schwartz, J. E., et of (1993) Substance abuse and onset of psychotic illness. Hospital and Community Psychiatry, 44, 567-571.

W. Hall National Drug and Alcohol Research Centre, The University of New South Wales, Sydney 2052, Australia

\section{Depressive delusions and the general election}

Sir: I would like to report the case of an 80year-old man, with no previous psychiatric or significant medical history, who six days after the landslide general election defeat of the Conservative Party developed depressive delusions that because of the Labour victory his investments were now worthless and his business was going to fail leaving him and his family penniless and forcing him to sell his house. He was agitated and extremely distressed by these ideas and believed his wife and adult daughter should kill themselves to avoid the shame of penury. He was in clear consciousness with no cognitive impairment. Mild symptoms of depression had started two weeks before with loss of energy and interests and sleep disturbance; these were treated with fluoxetine, to which he had shown no response. He was admitted to hospital but became acutely confused. Investigations showed hyponatraemia due to the syndrome of inappropriate antidiuretic hormone secretion, probably secondary to the fluoxetine. The delirium improved rapidly with discontinuation of fluoxetine and fluid restriction and he was left with mild depressive symptoms, but no further abnormal beliefs about the election. He made sense of his delusions by saying he had confused Tony Blair with Tony Benn! The political change going on around him clearly influenced the content of his delusions and may have affected the course of his illness. He was convinced he would not have been so hopeless about his future if there had been a hung parliament.

G. Yorston South Buckinghamshire NHS Trust, Shrubbery Road, High Wycombe, Buckinghamshire HPI3 6PS

\section{Role of GPs in service provision for people with schizophrenia}

Sir: Lang et al's (1997) interesting analysis is marred by a number of features. First, the sample data are not clearly defined. It would appear that the 193 patients who took part in the study were psychiatric outpatients. A recent review in my own practice found that more than one-third of those with schizophrenia were not known to the local psychiatric services.

Second, the difficulties in reviewing the entries in the case notes, although understandable, should have been dealt with by employing a GP researcher who would have been able to interpret the sometimes arcane way in which material is entered. Despite that, however, to recommend that there should be a standardised method for recording consultations in primary care is extraordinary. This shows a total lack of appreciation of the problems facing GPs. What is needed is hinted at later in the paper, where it is suggested that community psychiatric nurses (CPNs) should be involved. Our own practice is now undertaking a review of all patients by our locality attached CPN as a pilot to undertaking the same work throughout the six practices in our small locality. The aim of this review is to enter in the notes a care plan for each patient, with clear indications as to who the keyworker will be.

My other concern is that Lang et al fail to recognise a further major problem in primary care. At present, notes are kept separately by health visitors and by nurses in the majority of practices, with the result that there is no comprehensive primary care record. In the case of people with schizophrenia this can mean that contact for depot injections is undertaken by a nurse and recorded separately. In some cases the family will be supported by a health visitor, and again separate records may be kept.

This fragmentation, which is part of the inheritance of a divided primary care service, needs to be tackled along with developing seamless care for this group of patients. Lang et al's central finding that problem patients have a past history of drug misuse is, however, an important and helpful finding, although it is not clear whether this includes alcohol remaining as one of the problem areas in older patients with schizophrenia.

Lang, F. H., Johnetone, E. C. \& Murray, G. D. (1997) Service provision for people with schizophrenia: II. Role of the general practitioner. British journol of Psychiatry, 171, 165-168.

R. J. Simpson Bridge of Allan Health Centre, Fountain Road, Bridge of Allan FK9 4EU

Author's reply: Dr Simpson is right to draw attention to lack of contact which many people suffering from schizophrenia have with the local psychiatric services. In the companion paper to the one Dr Simpson comments upon (Lang et al, 1997) we clearly define our study populations. The 193 patients whom we studied had all had at least one admission to the Royal Edinburgh 